\title{
Interactions among sleep disordered breathing, obesity, and sleep duration
}

\author{
Kazuo Chin ${ }^{1}$
}

Published online: 16 March 2017

(C) Japanese Society of Sleep Research 2017

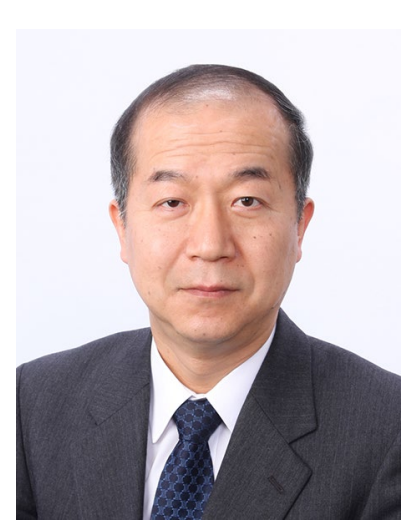

It was reported in the International Classification of Sleep Disorders (ICD-3) that there are seven groups of sleep disturbances: such as insomnia, sleep-related breathing disorders (SDB), central disorders of hypersomnolence, circadian rhythm sleep-wake disorders, parasomnias, sleeprelated movement disorders, and other sleep disorders, in which there are more than 60 sleep disorders. Seventeen of these disorders are classified as SDB, which includes obstructive sleep apnea, central sleep apnea, sleep-related hypoventilation disorders, etc. In addition to SDB, we should pay attention to the tendency toward the short sleep duration that has emerged in recent years, because short sleep duration has significant effects on general, cardiovascular, metabolic, and mental health, immune function, human performance, pain, and mortality. Recent data

Kazuo Chin

chink@kuhp.kyoto-u.ac.jp

1 Department of Respiratory Care and Sleep Control Medicine, Graduate School of Medicine, Kyoto University, Kyoto, Japan showed that during this 17-year period (1993-2010), survey reports of a diagnosis of sleep apnea increased 14.6fold, from 420,000 to 6.37 million per year $(P=0.0002)$ in the U.S [Namen AM, et al. Ann Am Thorac Soc. 2016; 13:2027-34]. In addition, more than 400,000 patients with obstructive sleep apnea (OSA) are treated by continuous positive airway pressure (CPAP) under the health insurance system in Japan and the number of CPAP patients increases by 40,000 per year in the previous 3 years. Thus, since the prevalence of SDB, short sleep duration, or the other sleep disturbances, such as insomnia, is high, we should promote research on the effects of not only SDB or short sleep duration itself but effects of combination of the systemic diseases and sleep-related problems on overall human health.

OSA is not only a factor in daytime hypersomnolence or other symptoms but in cardiovascular diseases and mortality. Obesity is a major risk factor for OSA and was confirmed as a main factor in cardiovascular diseases. Therefore, interaction between obesity and OSA should also be given attention. It is said that OSA affects parameters involved in the regulation of energy balance, including food intake, hormonal regulation of hunger/satiety, energy metabolism, and physical activity. Prior studies revealed a disruptive impact of OSA on energy metabolism, including humoral factors, and there is an emerging concept that OSA itself may in turn reinforce the obese state. Given the plausible reciprocal relationship between obesity and OSA, treating OSA with CPAP in theory could act against weight gain. However, recent studies revealed that CPAP therapy actually resulted in weight gain. In addition, the most recent study revealed a decrease of almost $5 \%$ in the basal metabolic rate (BMR) following 3 months of CPAP treatment during which physical activity and total caloric intake did not significantly change [Tachikawa R, et al. Am J Respir Crit Care Med. 2016; 194:729-38]. In that study, 
there were two types of patients with OSA: weight gainers and non-weight gainers. Among the parameters related to energy metabolism, increased caloric intake was a particularly significant predictor of weight gain. From that study, the authors' conclusion was that, although a reduction in BMR after CPAP predisposes to a positive energy balance, dietary intake and eating behavior had greater impacts on weight change. These findings highlight the importance of lifestyle modifications combined with CPAP. The higher brain center, which controls eating behavior, is a key factor in regulating body weight following CPAP treatment. That such eating behavior is predetermined or influenced by OSA should be investigated in a future study.
Thus, to know the interactions among sleep disordered breathing, obesity and sleep duration is an important issue in promoting good sleep and a healthy life extension. In addition, the interactions between these factors and the higher brain center may be the next important field of investigation. After uncovering the mystery of sleep, I hope that we will find the means toward ensuring good sleep that expands a healthy and satisfying life. 